\title{
List of churches by region
}

\section{Southern Scotland}

\section{Dumfries and Galloway}

Annan, Old Parish Church (72), Crossmichael, Parish Church (53), Dalswinton, Barony Church (139), Dumfries, Crichton Memorial Church (150), Durisdeer, Parish Church (47), Gretna, St Ninian's Catholic Church (165), Kirkcudbright, St Cuthbert's Parish Church (115), Kirkmaiden, Parish Church (39), Moffat, St Andrew's Parish Church (142), Newton Stewart, Penninghame St John's Parish Church (119), Southwick Church (146)

\section{Scottish Borders}

Channelkirk, Parish Church (93), Cranshaws, Parish Church (151), plate 21, Ettrick, Parish Church (99), Fogo, Parish Church (58), Galashiels, Old Parish Church and St Paul's (140), Greenlaw, Parish Church (44), Hoselaw Chapel (162), Innerleithen, Parish Church (128), Kelso, St Andrew's Scottish Episcopal Church (129), Ladykirk, St Mary's Parish Church (31), Lauder, Old Parish Church (43), Lyne, Parish Church (41), Stobo, Parish Church (9)

\section{East Central Scotland}

\section{Angus}

Brechin, Cathedral and Round Tower, (1), plate 1, Gardner Memorial Church (152) Cortachy, Parish Church (103), Forfar, Lowson Memorial Church (163), plate 23, Glamis, St Fergus' Parish Church (75), Kirriemuir, St Mary's Scottish Episcopal Church (154), Montrose, Old and St Andrew's Parish Church (73) 


\section{Clackmannanshire}

Alloa, St Mungo's Parish Church (94), Tillicoultry, St Serf's Parish Church (106)

\section{City of Dundee}

Dundee, Catholic Church of the Immaculate Conception (127), plate 19, St Andrew's Parish Church (64), St Peter's Free Church (113), Steeple and Town Churches (27, Fowlis Easter, Parish Church (23)

City of Edinburgh

Dalmeny, St Cuthbert's Parish Church (2), Edinburgh, Barclay Church (126), Broughton St Mary's Parish Church (98), Canongate Parish Church (46), Colinton Mains Church (175), Corstorphine Old Parish Church (26), Craigsbank Church (182), Duddingston Parish Church (10), Greyfriars Tolbooth and Highland Church (37), Nicolson Square Methodist Church (89), Palmerston Place Church (133) plate 20, Priestfield Church (138), Reid Memorial Church (168), St Andrew's and St George's Parish (68), St Cuthbert's Parish Church (147), St Giles' Cathedral (28), St John the Evangelist Scottish Episcopal Church (95), plate 17, St Mark's Unitarian Church (111), St Mary's Scottish Episcopal Cathedral (137), plate 22, St Mary Magdalene's Catholic Church (181), St Stephen's Church Centre (104), Leith, North Leith Parish Church (90), Portobello, St John the Evangelist's Catholic Church (157), Wilson Memorial United Free Church (169)

Fife

Anstruther, Parish Church (38), Burntisland, Parish Church (33), plates 10, 14, Culross, Abbey Church (12), plate 5, Dunfermline, Abbey (5), Abbey Church (97), plate 18, Dysart, Parish Church (132), Glenrothes, St Paul's Catholic Church (176), Kilmany, Parish Church (69), Leuchars, St Athernase's Parish Church (8), plate 4, Limekilns, Parish Church (101), St Andrews, Holy Trinity Parish Church (20), St Monan's, Parish Church (19), plate 7

\section{East Lothian}

Dirleton, Parish Church (35), East Saltoun, Saltoun Parish Church (81), Haddington, St Mary's Parish Church (17), Inveresk, St Michael's Parish Church (83), Port Seton, Chalmers Memorial Church (156), Yester, Parish Church (48) 


\section{Midlothian}

Roslin, Rosslyn Chapel (St Matthew's Scottish Episcopal Church) (24)

\section{West Lothian}

Linlithgow, St Michael's Parish Church (21), plates 8, 11, St Peter's Scottish Episcopal Church (167), Livingston, St Andrew's Catholic Church (183), plate 25, Torphichen, Parish Church (60)

\section{Perth and Kinross}

Alyth, Parish Church (117), Collace see Kinrossie, Dunkeld, Cathedral (18), Kinrossie, Collace Parish Church (88), Perth, St John's Parish Church (22), St Leonard's-in-the-Fields and Trinity Parish Church (143), St Ninian's Scottish Episcopal Cathedral (121) Scotlandwell, Portmoak Parish Church (107)

\section{Stirling}

Dunblane, St Blane's Cathedral (11), Lecropt, Parish Church (100), Stirling, Church of the Holy Rude (29)

\section{West Central Scotland}

\section{East Ayrshire}

Catrine, Parish Church (74), Fenwick, Parish Church (40), Galston, St Sophia's Catholic Church (144), Kilmarnock, Old High Parish Church (52), Muirkirk, Parish Church (87)

\section{North Ayrshire}

Ardrossan, St Peter-in-Chains Catholic Church (171), Cathedral of the Isles see Millport, Kilbirnie, Old Parish Church (25), plate 9, Saltcoats, St Cuthbert's and South Beach Parish Church (160), Shiskine, St Molio's Church (145)

\section{South Ayrshire}

Auchincruive, St Quivox Parish Church (34), Ayr, Old Parish Church (42), Irvine, Old Parish Church (65), Symington (Ayrshire), Parish Church (6), Troon, Our Lady and St Meddan's Catholic Church (161)

\section{East Dunbartonshire}

Baldernock, Parish Church (77) 
West Dunbartonshire

Clydebank, St Joseph's Catholic Church (184)

City of Glasgow

Eastbank Church (155), Kelvinside Hillhead Parish Church (135), Queen's Park Baptist Church (134), Renfield St Stephen's Church (123), St Andrew's Catholic Cathedral (91), plate 16, St Columba's Catholic Church (173), plate 24, St George's Tron Parish Church (85), St Mungo's Cathedral (3), plate 2, St Vincent Street-Milton Free Church (124), Wellington Church (141)

Inverclyde

Greenock, Old West Church (166), St Luke's Parish Church (120), Wellpark Mid Kirk (61)

North Lanarkshire

Airdrie, St Margaret's Catholic Church (116), Coatbridge, St Andrew's Parish Church (118), St Patrick's Catholic Church (149), Cumbernauld, St Mungo's Parish Church (180), Kilsyth, St Patrick's Catholic Church (178), Kirk o'Shotts, Parish Church (96)

South Lanarkshire

Bothwell, Parish Church (108), Dalserf, Parish Church (49), plate 12, East Kilbride, St Bride's Catholic Church (177), Hamilton, Old Parish Church (51)

Renfrewshire

Paisley, Abbey (7), plate 3, Oakshaw Trinity (59), plate 15, St Matthew's Church of the Nazarene (159), Thomas Coats Memorial Baptist Church (148), Wallneuk North Church (164), Renfrew, Old Parish Church (125)

East Renfrewshire

Newton Mearns, Mearns Parish Church (56)

\section{North East Scotland}

City of Aberdeen

Greyfriars John Knox Church (153), King's College Chapel (30), plate 13, St Andrew's Scottish Episcopal Cathedral (92), St Machar's Cathedral (16), plate 6, St Mary's Church, King Street (172), St Nicholas Parish Church (55) 


\section{Aberdeenshire}

Arbuthnott, St Ternan's Parish Church (13), Bourtie, Parish Church (82), Braemar, St Margaret's of Scotland Scottish Episcopal Church (158), Echt, Parish Church (80), Huntly, St Margaret's Catholic Church (110), Peterhead, Old Parish Church (84)

\section{Moray}

Cullen, Old Kirk (14), Dyke, Parish Church (66), Elgin, St Giles' Parish Church (105), Fochabers, Bellie Parish Church (78), Gordon Scottish Episcopal Chapel (109), Tynet (Mill of), St Ninian's Catholic Chapel (57)

\section{Highlands and Islands}

\section{Argyll and Bute}

A'Chleit (Kintyre), Killean and Kilchenzie Parish Church (70), Achnaba, Ardchattan Parish Church (112), Bowmore, Kilarrow Parish Church (63), Campbeltown, Highland Parish Church (79), Dalmally, Glenorchy Parish Church (86), Dunoon, St John's Church (136), Glendaruel (Clachan of), Kilmodan Parish Church (67), Inveraray, Glenaray and Inveraray Parish Church (76), Millport, The Scottish Episcopal Cathedral of the Isles (122)

\section{Highland}

Ardgay see Croick, Cawdor, Parish Church (36), Croick, Parish Church (102), Dingwall, Free Church (131), Dornoch, Cathedral (15), Dunnet, Parish Church (32), Fort William, Catholic Church of the Immaculate Conception (170), Golspie, St Andrew's Parish Church (50), Inverness, Old High Kirk (62), St Andrew's Scottish Episcopal Church, St Mary's Catholic Church (114), Tongue, St Andrew's Parish Church (45)

\section{Orkney}

Kirkwall, St Magnus' Cathedral (4), Lamb Holm, Italian Chapel (174)

\section{Shetland}

Lunna, Lunnasting Parish Church (54), Tingwall, St Magnus Parish Church (71)

Western Isles

Garrynamonie, Our Lady of the Sorrows Catholic Church (179) 